\title{
New neurotechnologies for the diagnosis and modulation of brain dysfunctions
}

This is a major review article to acquaint psychologists with new neurotechnologies for the diagnosis and modulation of brain abnormalities. While psychometrics measures brain functions in terms of behavioral parameters, a recently emerged branch of neuroscience called neurometrics relies on measuring the electrophysiological parameters of brain functioning. There are two approaches in neurometrics. The first relies on the spectral characteristics of spontaneous electroencephalograms (EEG) and measures deviations from normality in EEG recorded in the resting state. The second approach relies on event-related potentials (ERPs) that measure the electrical responses of the brain to stimuli and actions in behavioral tasks.
The present study reviews recent research on the application of ERPs for the discrimination of different types of brain dysfunction. Attention deficit-hyperactivity disorder (ADHD) is used as an example. It is shown that the diagnostic power of ERPs is enhanced by the recent emergence of new methods of analysis, such as independent component analysis (ICA) and low resolution electromagnetic tomography (LORETA).

\section{KEY WORDS}

event-related potentials (ERPs); attention deficit-hyperactivity disorder (ADHD); electroencephalogram (EEG)

ORganizations - 1: Institute of the Human Brain, Russian Academy of Sciences, St. Petersburg, Russia·2: Institute of Psychology, Norwegian University of Science and Technology, Trondheim, Norway 3: Chair of Neuropsychology, Andrzej Frycz Modrzewski Cracow University, Cracow, Poland, 4: Center for Cognition and Communication, New York, NY, USA, 5: Praxis für Kind, Organisation und Entwicklung, Chur, Switzerland authors' Contribution - A: Study design - B: Data collection - C: Statistical analysis - D: Data interpretation . E: Manuscript preparation · F: Literature search · G: Funds collection

CORRESPonding AUthor - Prof. Juri Kropotov, Institute of the Human Brain, Russian Academy of Sciences, Academica Pavlova 12 a, 197376 S. Petersburg, Russia, e-mail: yurykropotov@yahoo.com

to Cite this ARTicle - Kropotov, J. D., Pąchalska, M., \& Mueller, A. (2014). New neurotechnologies for the diagnosis and modulation of brain dysfunctions. Health Psychology Report, 2(2), 73-82. DOI: 10.5114/hpr.2014.43913 


\section{INTRODUCTION}

Suppose a boy comes to the door of a neurologist, psychiatrist or any health care practitioner. His behavior looks like typical attention deficit-hyperactivity disorder (ADHD): he is extremely inattentive, impulsive and hyperactive. He performs poorly in continuous performance tasks. Recent research in the neurophysiology of ADHD shows that there are several reasons why the boy behaves in this way.

Juri D. Kropotov, Maria Pąchalska, Andreas Mueller

A patient may have:

1) a focus in his cortex, which without any overt symptoms of epilepsy impairs information processing and, consequently, mimics attention deficit (see: Duane, 2004; Pąchalska, Kaczmarek \& Kropotov, 2014; Socanski, Herigstad, Thomsen, Dag \& Larsen, 2010; Vendrame et al., 2010);

2) a lack of overall cortical activation due to dysfunction of the ascending reticular system of the brain stem (Sergeant, 2005; Conzelmann et al., 2014);

3) genetically determined hyperactive frontal lobes with elevated beta activity (Clarke, Barry, McCarthy \& Selikowitz, 2001; Clarke et al., 2013). Behaviorally, children with excess beta activity have been found to be more moody and prone to temper tantrums as well as showing increased delinquent behavior (Clarke et al., 2011);

4) dysfunction of the prefrontal-striato-thalamic system due to a structural abnormality (Castellanos, 2002; Kieling, Goncalves, Tannock \& Castellanos, 2008); or an increase of dopamine reuptake dopamine transporters in the striatum (Krause, Dresel, Krause, la Fougere \& Ackenheil, 2003);

5) dysfunction in the anterior cingulate gyrus (Albrecht et al., 2008) which may produce anxiety, emotional instability and hyperactivation.

By knowing which brain dysfunction is associated with the symptoms of ADHD, a clinician can suggest an individualized treatment. The choice of treatment can be either a medication using a dopamine reuptake inhibitor (such as Ritalin [methylphenidate]), or a medication using a noradrenaline reuptake inhibitor (such as Straterra), or the patient can respond well to neurofeedback (NFB), or the optimal treatment may be transcranial direct current stimulation (for a review see Kropotov, 2009), or the patient may simply respond to $\gamma$-aminobutyric acid (GABA) agonists which "shut down" the epileptic focus.

Recent research shows that the above-mentioned dysfunctions are associated with specific patterns in spontaneous and evoked electrical potentials, recorded from the head by multiple surface electrodes. Spontaneous and evoked electric potentials recorded from the head provide reliable brain markers of the brain function and dysfunction, while EEG-based biofeedback and weak electric currents are capable of normalizing electroencephalograms (EEG) and modifying behavior.

\section{NEUROMETRICS AS AN ADJUNCT TO PSYCHOMETRICS}

Psychologists rely on psychometrics to gauge personality, pathology, motivation, and learning difficulties. But despite built-in lie scales, split half designs and a host of other clever statistical manipulations, psychometric measurements are still represented by behavioral data gained from self or other reports.

A recently emerged science, called neurometrics, relies on measuring the underlying organization of the human brain's electrical activity. According to E. Roy John, an outstanding American neurobiologist who coined the term in the 1970s, neurometrics is "a method of quantitative EEG that provides a precise, reproducible estimate of the deviation of an individual record from normal. This computer analysis makes it possible to detect and quantify abnormal brain organization, to give a quantitative definition of the severity of brain disease, and to identify subgroups of pathophysiological abnormalities within groups of patients with similar clinical symptoms" (John, 1990).

Entrepreneurs began to take notice of the potential of neurometrics in the late 1980s. Three commercial systems were sequentially registered in 1988 (Neurometric Analysis System, based on the University of New York normative data, published by John et al. [John, 1977]), in 2004 (Neuroguide Analysis System, based on the University of Maryland normative data, published by Thatcher et al. [Thatcher, 1998]), in 2005 (BRC Software Product, based on the normative data collected internationally in several laboratories, published by Gordon, Cooper, Rennie, Hermens \& Williams, 2006). Each of these devices represents software which is capable of comparing a subject's EEG data to a normative database, thus giving clinicians a tool for measuring the patient's variance from the normal.

\section{NORMATIVE DATABASES IN ELECTROENCEPHALOGRAMS}

There are many normative neuroscience databases which include computed tomography (CT), magnetic resonance imaging (MRI), positron emission tomography (PET) and other datasets. However, quantitative EEG and event-related potential (ERP) normative databases play a critical role among many others in clinical practice for the following reasons.

1. We are entering a new era of psychiatry and neurology. Long awaited, the fifth revised edition of The Diagnostic and Statistical Manual of Mental Disorders (DSM-V) will became the main reference for psychiatrists and neurologists upon its release in 2013. The primary focus of the new revision is to classify brain disorders according to their biologi- 
cal markers - endophenotypes. The new approach assumes that a psychiatric diagnosis is made not only from behavior, but also from the knowledge of which brain system is impaired. The only dynamic (on a millisecond time scale) parameters of the brain function are provided by EEG and magnetoencephalography (MEG). At the moment, MEG machines are too expensive, while EEG machines, as they are inexpensive, currently remain the primary choice for clinicians (Kropotov, 2014).

2. We are facing a renaissance in EEG. On the one hand, the renaissance is associated with obtaining new knowledge regarding the neuronal mechanisms for the generation of alpha, theta and beta oscillations in spontaneous EEG as well as regarding the functional meaning of different waves in event-related potentials (for a review see Kropotov, 2009).

3. On the other hand, the renaissance is associated with the development of new methods of analysis. These methods (e.g. the decomposition of EEG and evoked responses into independent components, low resolution electromagnetic tomography LORETA) were initiated in laboratory settings only ten years ago (see Makeig, Bell, Jung \& Sejnowski, 1996; Pascual-Marqui, 1999).

There is an urgent need to introduce these new methods into clinical practice. Unfortunately, none of the existing normative databases use the newly developed technologies. This flaw of the current databases is resolved in a new database constructed on the methodology developed in the Human Brain Institute (HBI) of the Russian Academy of Sciences and the Institute for Experimental Medicine of the Russian Medical Academy of Sciences. The methodology is presented in a series of papers and summarized in the book recently published by Academic Press (Kropotov, 2009). A database called HBI (The Human Brain Index) reference database is now used in many scientific centers in Europe and the USA.

\section{ELECTROENCEPHALOGRAM ENDOPHENOTYPES OF BRAIN DISORDERS}

We focus on the most common and, undoubtedly, the most controversial disease - attention deficithyperactivity disorder. The first theoretical attempt to introduce endophenotypes as neuroscience-based markers of ADHD was done by Xavier Castellanos and Rosemary Tannock (see: Castellanos \& Tannock, 2002). The authors proposed three endophenotypes that would correspond to the causes of ADHD: 1) a specific abnormality in reward-related circuitry that leads to shortened delay gradients, 2) deficits in temporal processing that result in intra-subject internal variability, 3) and deficits in working memory.
In practice, several attempts were made to discriminate the ADHD population from healthy subjects. These attempts explore various imaging techniques including PET, MRI and functional magnetic resonance imaging (fMRI). Here we discuss only studies in the field of EEG. In general terms, spectral characteristics of EEG are considered to be good indicators of metabolic activity within the cortex. An excessive slow activity and a lack of beta activities in local EEG indicates the low metabolic activity of the underlying cortical area (Cook, O’Hara, Uijtdehaage, Mandelkern \& Leuchter, 1998).

\section{ELECTROENCEPHALOGRAM SPECTRA IN ADHD}

The most commonly used form of EEG analysis in studies of ADHD has been the calculation of absolute and relative power estimates. The research in this field is enormous. Simply a search for the key words "ADHD and EEG" gives 667 papers from 1970 to the present time. In this paper we will mention only the most critical papers.

Chabot and Serfontein (1996) reported EEG differences in 407 ADHD children compared to a normative database. Children with ADHD had an increase in absolute and relative theta, primarily in the frontal regions and at the frontal midline. Clarke, Barry, McCarthy and Selikowitz (1998) carried out the first study of EEG differences between children with different DSM-IV types, comparing 20 ADHD combined type, 20 ADHD inattentive type and 20 control subjects, using an eyes-closed resting condition. The ADHD groups had increased power levels across all sites in absolute and relative theta, and reductions in the relative alpha and beta. In a follow-up study with larger independent subject groups Clarke et al. (2001) found ADHD children to have increased absolute and relative theta, and decreased relative alpha and beta, and these effects differentiated ADHD combined type from ADHD inattentive type.

Bresnahan, Anderson and Barry (1999) performed the first study to investigate the EEG profiles of adult ADHD subjects, using 3 age groups: children, adolescents and adults, with age- and sex-matched controls. The results indicated that absolute and relative theta activity remained elevated through adolescence into adulthood.

\section{RATIO COEFFICIENTS}

The ratio between power in different frequency bands has been used to evaluate changes in the EEG that occur due to normal maturation (Matousek \& Petersen, 1973) and as a measure of cortical arousal (Lubar, 1991). Matousek, Rasmussen and Gilberg (1984) found
Diagnosis and modulation of brain dysfunctions 
Juri D. Kropotov, Maria Pąchalska, Andreas Mueller that the theta/alpha ratio was a good predictor of group differences between children with MBD, ADD and control subjects. Janzen, Graap, Stephanson, Marshall and Fitzsimmons (1995) reported that children with ADHD had a higher theta/beta ratio than control subjects. Monastra et al. (1999) calculated the theta/beta ratio from 482 individuals aged 6-30 years old and found that the theta/beta ratio was higher in ADHD subjects than control subjects. Clarke et al. (2001) found that both the theta/alpha and theta/beta ratios can differentiate between groups of normal children and children with ADHD. Further, the ratio distinguished adults who met ADHD criteria from those with some symptoms of the disorder who failed to meet those criteria (Bresnahan \& Barry, 2002), indicating some specificity for this marker in ADHD. Sensitivity of the inattentive index (theta beta ratio) was found to be $86 \%$, and specificity $98 \%$ (Monastra et al., 1999).

\section{EVENT-RELATED POTENTIALS IN ADHD}

Since the early 1970s (e.g. Satterfield, Cantwell, Lesser \& Podosin, 1972; Buchsbaum \& Wender, 1973; Saletu, Saletu \& Itil, 1973) ERP studies have revealed much about information processing in ADHD. Some of these studies focused on tasks testing the functioning of the auditory and visual attention systems, with others examining so-called executive functions

Typical paradigms used in ERP studies of ADHD include auditory or visual selective attention tasks, combined-modality selective attention tasks, 2- or 3-tone oddball tasks, S1-S2 tasks, go/no-go tasks and stop-signal tasks. A literature search for the two keywords "ADHD and Event Related Potentials" in PubMed gave 382 papers published within the period from 1970 to the present time (Pąchalska et al., 2014).

Here we focus only on the late positive complex in the visual modality in ERP studies. The visual P3 component has been reported to differentiate clinical from control children. For example, P3 amplitude was reduced in ADHD subjects in a letter sequence oddball task (Holcomb, Ackerman \& Dykman, 1985), in easy and difficult versions of a letter oddball task, with larger differences being evident in the hard task (Lubar, 1991), during classification and oddball tasks (Robaey, Breton, Dugas \& Renault, 1992), and during several CPT studies (Klorman, Salzman, Pass, Borgstedt \& Dainer, 1979; Overtoom et al., 1998) and other visual selective attention tasks (Jonkman et al., 1997).

In recent years there has been a shift of focus away from attention-based accounts of ADHD deficits, towards 'executive functions' (e.g. Barkley, 1997). Within this context, the focus has been on the psychological process of inhibition, and its role in the behavioral manifestations of ADHD. Specific paradigms believed to access this process, such as the go/no-go task and the stop-signal task, have been utilized.

Event-related potentials recorded during inhibitory processing typically contain a negative component approximately $200 \mathrm{~ms}$ after the onset of the inhibition-evoking stimulus, which is believed to reflect a frontal inhibition process (Kopp, Mattler, Goertz \& Rist, 1996). Research with the oddball task suggests deficits in inhibitory processing in ADHD. For example, a reduced frontal N2 peak in ADHD compared to control subjects was found, indicating an atypical frontal inhibition process in ADHD (Johnstone \& Barry, 1996). Similar results have been reported in inhibition-specific tasks. Using the stop-signal task with an auditory stop signal, frontal N2 amplitude to the stop stimulus was dramatically reduced in ADHD (Pliszka, Liotti \& Woldorff, 2000).

Here we present some results of our own multicentre study, which was conducted within the framework of the COST B 27 initiative. This initiative was sponsored by the European Commission Research Foundation and included 5 countries: Switzerland (Andreas Mueller and his group), Austria (Michael Doppelmayr and his group), Norway (Stig Hollup and his group), Macedonia (Jordan Pop-Jordanov and his team), and Russia (Juri Kropotov and his lab). In particular, the study included the recordings of 150 ADHD children (24 girls) aged from 7 to 12 years. Below we are presenting the results of a comparison between two age-matched groups of healthy subjects (taken from the HBI reference normative database) and ADHD children recorded under the same task conditions. All subjects and patients participated in the same two-stimulus GO/NO-GO task.

Seven independent components, constituting $87 \%$ of the signal, were separated from the collection of ERPs recorded in response to GO and NO-GO stimuli. Four of them are presented in Figure 1. As one can see, only one component significantly (with the size effect of 0.43) discriminates the ADHD group from the control healthy group. This component is generated in the premotor cortex. Its reduction in ADHD reflects functional hypoactivation of the premotor area in the inhibitory control in children with attention deficit.

\section{NEUROFEEDBACK IN ADHD}

Fifty years of using psycho-pharmacology for the treatment of brain disorders brought some dissatisfaction and controversy. One part of the controversy is associated with the side effects induced by the regular consumption of psychoactive drugs. For example, in ADHD, the most common side effects of psychostimulants include headaches, abdominal pain, appetite suppression, irritability, insomnia, and hyperten- 

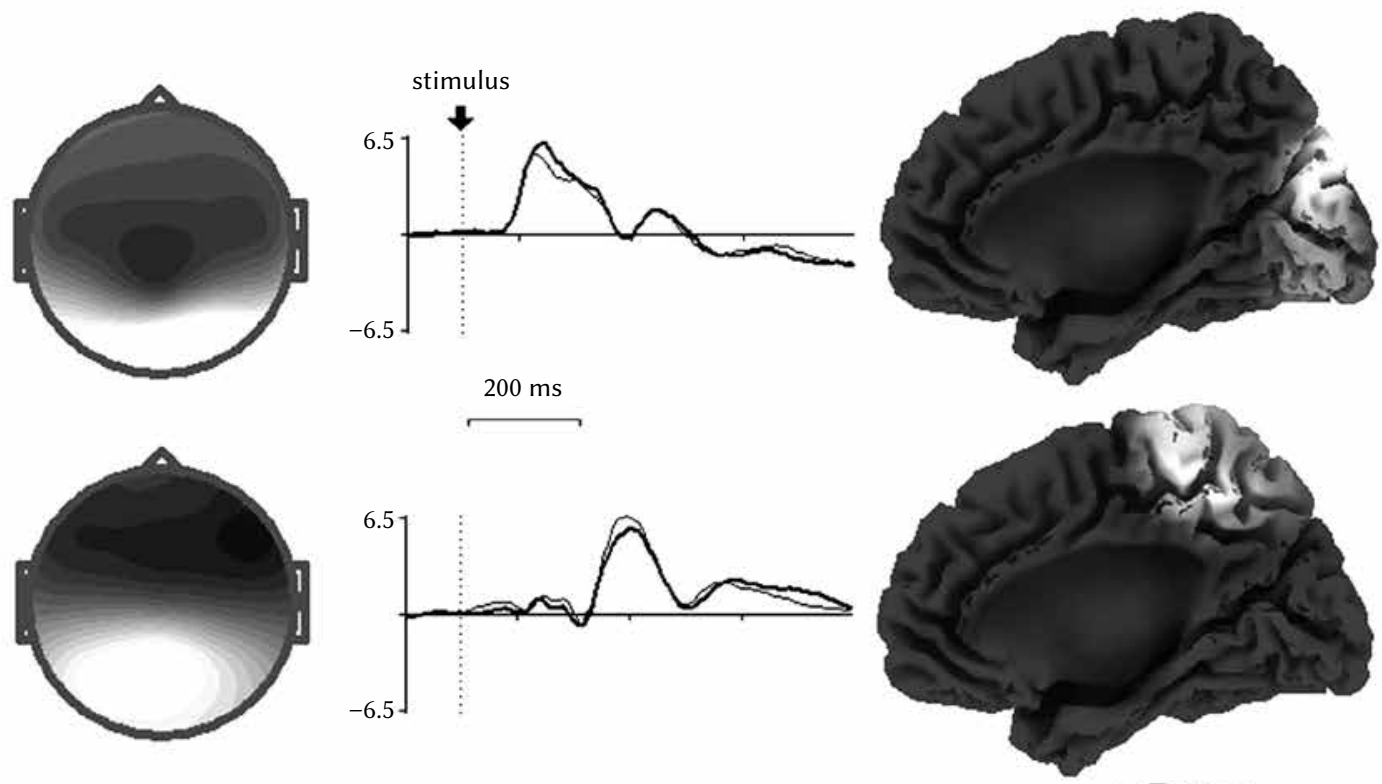

Diagnosis

and modulation

of brain

dysfunctions
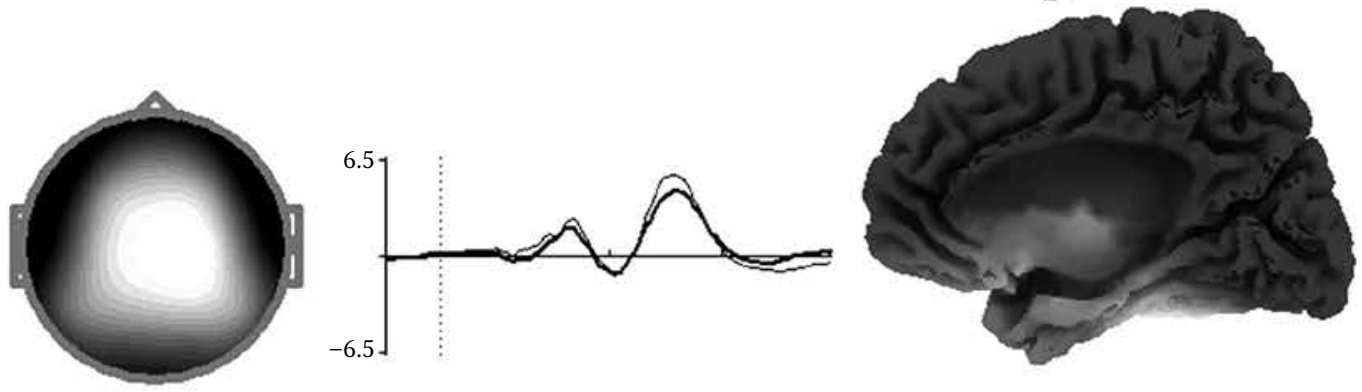

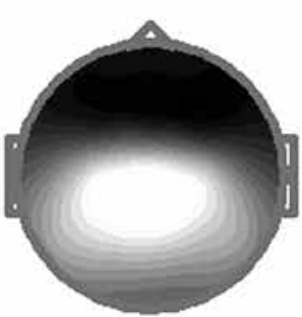

Topography healthy

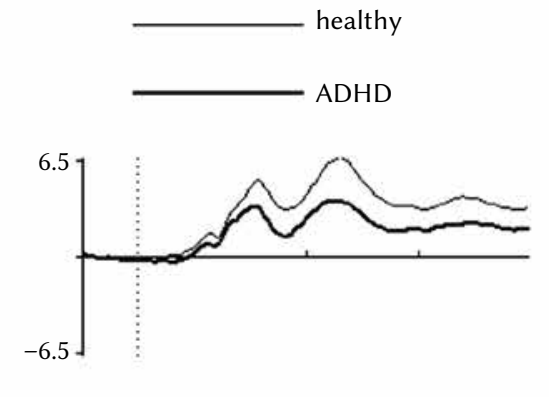

Time course

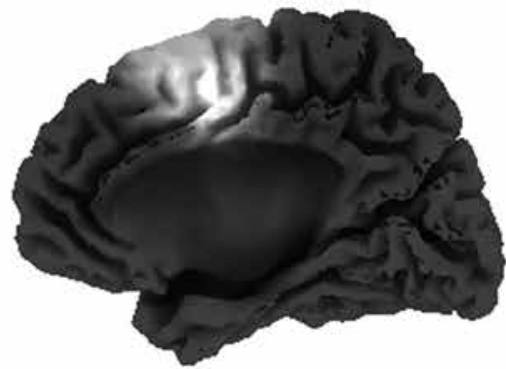

sLORETA image

Figure 1. Independent components extracted from the collection $(N=300)$ of ERPs of the group of ADHD children and healthy controls. ERPs were recorded in the cued GO/NO-GO task (Kropotov \& Mueller, 2009). Left - topography of the components. Right - sLORETA images of the topographies. Middle - time course of the components to NO-GO stimuli in healthy (thin line) and ADHD (thick line) children aged 7-12 years.

sion. The other part of the controversy is associated with a failure to find a genotype for a certain disease. The recent attempts of medical genetics have indicated that ADHD as with most psychiatric disorders does not follow a simple Mendelian rule and that no single gene could be attributed to a single disorder.

This led some scientists to introduce the concept of endophenotypes as biological markers of disease that are non-molecular but closer to the genotype than a behaviorally defined classification of diseases. One of such endophenotypes of ADHD is shown in Figure 1. It represents the functional hypoactivation of the premotor cortex of $\mathrm{ADHD}$ children during NO-GO trials. This ERP-based endophenotype fits the increased theta activity and decreased beta activity over the central-frontal regions observed in studies of spontaneous EEG oscillations in an ADHD population. 
Juri D. Kropotov, Maria Pąchalska, Andreas Mueller
Nowadays we know at least three different interventions that would activate the frontal-central areas of the cortex. They are psychostimulants, neurofeedback and transcranial direct current stimulation.

Historically, the fact that $30 \%$ of the ADHD population could not be treated by psychostimulants motivated researchers to search for alternative forms of treatment. The rationale for EEG biofeedback was derived from substantial neurophysiological research and quantitative electroencephalography (QEEG) assessment in the ADHD population. One of the leading scientists in this field, Barry Sterman, in his review (Sterman, 1996) indicated that "variations in alertness and behavioral control appear directly related to specific thalamocortical generator mechanisms and that such variations are evident in distinctive EEG frequency rhythms that emerge over specific topographic regions of the brain”. He hypothesized that neuropathology (such as ADHD) could alter these rhythms and that EEG feedback training directed at normalizing these rhythms may yield sustained clinical benefits.
Retrospectively, based on extensive research during the last decade we now recognize the existence of QEEG sub-types in ADHD and understand the need for different neurofeedback protocols to correct QEEG abnormalities in ADHD sub-types, but historically some of the protocols during the first years of the neurofeedback era were obtained empirically. Most of the protocols use the conventional EEG in a frequency range higher than $0.1 \mathrm{~Hz}$, while EEG at lower frequencies was used in studies of a German group at the University of Tuebingen (Strehl et al., 2006).

Here we present the data of our studies which applied a so-called relative beta training protocol (Kropotov et al., 2005). Figure $2 \mathrm{~A}$ schematically represents a comparison between the power spectra of one of the ADHD children subtypes and normal controls. EEG recording is conducted over the $\mathrm{Fz}-\mathrm{Cz}$ area and is shown in Figure 2B. As one can see, this ADHD subtype is characterized by excessive EEG power in the lower (theta) band and a lack (in comparison to norms) of EEG power in the beta frequency band.
A

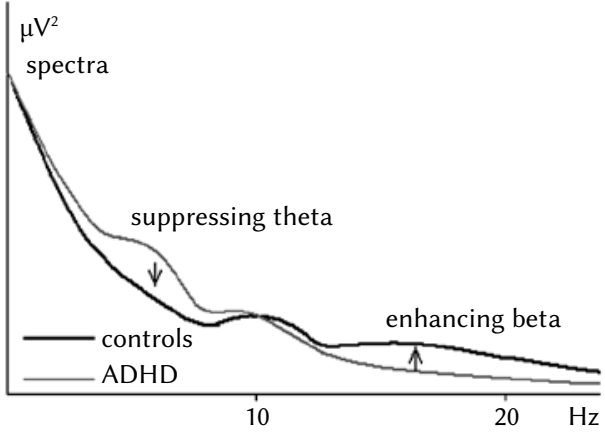

C

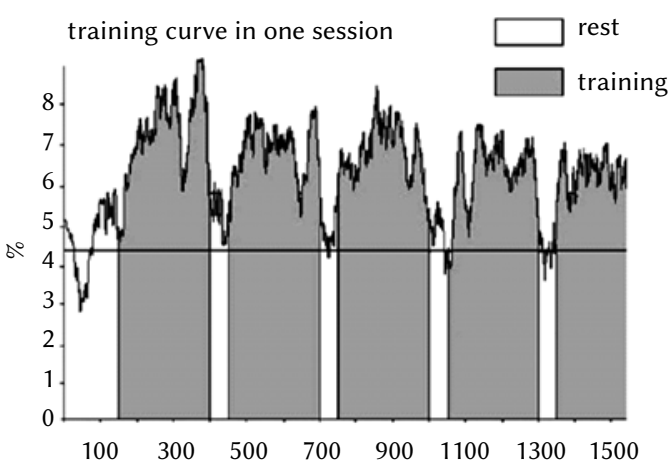

B

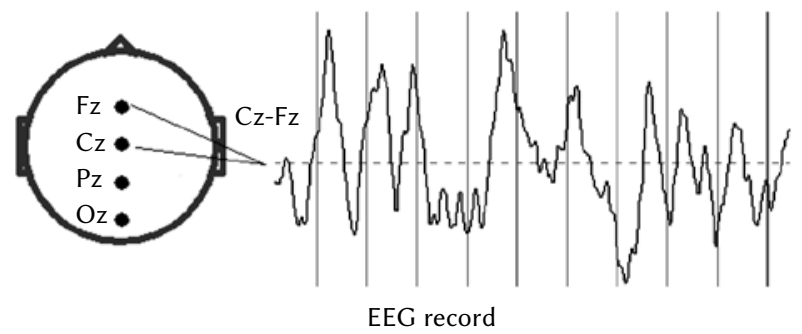

D

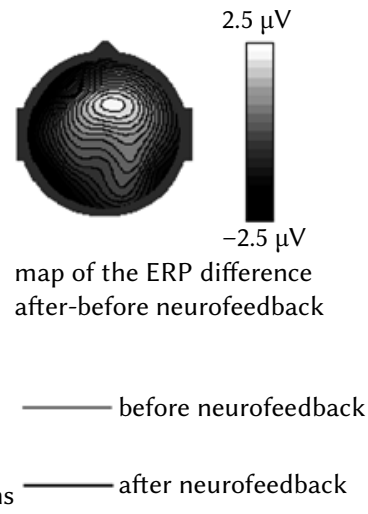

Figure 2. Effect of relative beta neurofeedback protocol on ERPs in the cued GO/NO-GO task of ADHD children. A) Schematic presentation of EEG spectra for a group of healthy controls (thick line) and a group of ADHD children (thin line) with excessive theta/beta ratio. X-axis - frequency. Y-axis - EEG spectra at Cz. B) An EEG fragment of an ADHD patient at Fz-Cz bipolar montage. C) Time dynamics (X-axis in s) of the neurofeedback parameter (Y-axis) in a neurofeedback session. White area - resting period. Gray area - training period. D) Increase of amplitude of the NO-GO ERP wave in the ADHD group after 20 sessions of relative beta training. Thin line - pre-, thick line - post-training. On the right - the map of the post-pre difference wave. Adapted from Kropotov et al. (2005). 
This pattern of deviation from normality results in a decrease in the relative beta activity.

In our studies the EEG was recorded bipolar from the central and frontal electrodes (Figure 2B). A typical training session included 20 minutes of relative beta training. The biofeedback procedure consisted of the following computations: the power spectrum was calculated for a 1-s epoch every $250 \mathrm{~ms}$ using fast Fourier transformation. Visual feedback was provided by a bar against a background on a computer screen. The height of the bar followed the dynamics of the biofeedback parameter. The patient's task was to keep the bar above a threshold determined at the pre-training 2-minute interval.

In addition to the simple visual feedback, a socalled video mode was used. In this mode, the biofeedback parameter controlled the level of noise generated by a separate electronic unit. The amplitude of the noise was maximal if the biofeedback parameter was minimal, and decreased gradually to zero while the parameter approached a threshold. The noise was mixed with the video signal of the video player and was fed to a TV set. Thus the patient actually controlled the quality of the picture on the screen by his/her brainwaves: when the biofeedback parameter was higher than the threshold, the picture on the screen was clear, otherwise the TV picture was blurred by the noise.

Usually during the first five to eight sessions, patients performed training in the simple visual mode with the bar to be able to get a feeling for the procedure. Then training in the video mode started. The dynamics of the biofeedback parameter (training curve) were obtained for each patient and for each session. Figure 2C shows a typical training curve for a single patient taken at the $15^{\text {th }}$ session. One can see that the patient was able to elevate the parameter during periods of training while the parameter dropped to the pre-training level during rest periods.

It should be noted that not all patients were able to reliably elevate the relative beta activity even after 10-20 sessions. Seventy-one patients out of $86(82.5 \%)$ were assigned to the good performance group. To test the functioning of the executive system, ERPs in the auditory two-stimulus GO/NO-GO task were recorded before and after all sessions of neurofeedback. Event-related potentials to NO-GO curves superimposed on each other in "before" and "after" recordings are presented in Figure 2D. One can see enhancement of the positive component at the frontal leads after 20 sessions of the relative beta training. The map of grand average ERP differences is presented in Figure 2D, right. It should be noted that the relative beta training does not change early (with latencies of 80-180 ms) components of ERPs but leads to significant enhancement of later positive components. Thus, our data indicate that relative beta training does not affect sensory information processing in the human brain, while it significantly changes the functioning of the executive system reflected in the late ERP components.

Theoretically, our protocol differs from conventional protocols, because elevation of the biofeedback parameter in our study could be achieved by increasing beta power, and/or by decreasing theta as well as alpha power. However, as the results of our study indicate, the application of the relative beta protocol turns out to be as effective as conventional protocols. Moreover, according to parents' assessment by SNAP-IV, neurofeedback significantly improved behavior, as reflected in the corresponding changes of indexes of inattention and impulsivity.

\section{TRANSCRANIAL DIRECT CURRENT STIMULATION IN ADHD}

In our laboratory at the Institute of the Human Brain in Saint Petersburg we are using transcranial direct current stimulation (tDCS) for correcting symptoms of ADHD in addition to the neurofeedback training. The history of the development of this method goes back to the 1960s when cathodic micro-polarization was first applied to switch off the pathological rhythmic firing of neurons in the subcortical structures of patients with Parkinson's disease. Several years of experiments on dogs and cats in the 1970s confirmed that the application of negative DC potentials to the electrodes implanted in the deep structures induced suppression of the impulse activity of neurons located near the electrodes, while the application of anodic currents produced an opposite effect (Vartanyan et al., 1980). After experiments on dogs and cats the method was introduced into clinical practice for treatment of different neurological dysfunctions in adults. Recently, we have started to use low anodic current stimulation for ADHD children.

In our studies (Kropotov, Chutko, Iakovenko \& Grin'-Iatsenko, 2002) an anode electrode was placed over the frontal lobe (F7 and F8 site) while the cathode was placed near the mastoids. The direct current was in the range $700-1000 \mu \mathrm{A}$ and tailored individually so that the patients could not feel the current. Sessions of tDCS lasted for 20 minutes and were repeated every 2-3 days, with the total number of sessions being 7 .

Neural circuits are pruned from the womb on - some reinforced by repetitive patterns (mother's voice, the smell of food, etc.) and others dropped from the connecting network. Life's experience shapes and progressively refines brainwave circuits so that, like fingerprints, brain waves are unique. But there are similarities on which QEEG databases rely. For example, the neural signatures of developmental processes that have been disrupted have a similar pattern.

Dyslexics will show a lower amplitude of "task beta" in the language circuits used for reading for ex-
Diagnosis and modulation of brain dysfunctions 
Juri D. Kropotov, Maria Pąchalska, Andreas Mueller ample, and depression is associated with a high amplitude of alpha in the left prefrontal cortex.

Quantitative electroencephalogram (QEEG) is likely to be a psychologist's best partner in looking through this window to the brain, as the equipment becomes, as was the case with computers, cheaper, smaller and easier to use. Courses in the theory and practice of neurotherapy are increasingly common in the United States and in the UK. The Society for Applied Neuroscience is organizing a Master's degree program for professionals who wish to add this therapy to their practice.

\section{REFERENCES}

Albrecht, B., Brandeis, D., Uebel, H., Heinrich, H., Mueller, U. C., Hasselhorn, M., Steinhausen, H. C., Rothenberger, A., \& Banaschewski, T. (2008). Action monitoring in boys with attention-deficit/ hyperactivity disorder, their nonaffected siblings, and normal control subjects: evidence for an endophenotype. Biological Psychiatry, 64, 615-625.

Barkley, R. A. (1997). Behavioral inhibition, sustained attention, and executive functions: Constructing a unifying theory of ADHD. Psychological Bulletin, 121, 65-94.

Bresnahan, S., Anderson, J., \& Barry, R. (1999). Agerelated changes in quantitative EEG in attention deficit disorder. Biological Psychiatry, 46, 1690-1697.

Buchsbaum, M., \& Wender, P. (1973). Averages evoked responses in normal and minimally brain dysfunctioned children treated with amphetamine. Archives of General Psychiatry, 29, 764-770.

Castellanos, F. X. (2002). Anatomic magnetic resonance imaging studies of attention-deficit/hyperactivity disorder. Dialogues Clinical Neuroscience, 4, 444-448.

Castellanos, F. X., \& Tannock, R. (2002). Neuroscience of attention-deficit/hyperactivity disorder: the search for endophenotypes. Nature Reviews Neuroscience, 3, 617-628.

Clarke, A., Barry, R., McCarthy, R., \& Selikowitz, M. (1998). EEG analysis in Attention-Deficit/Hyperactivity Disorder: a comparative study of two subtypes. Psychiatry Research, 81, 19-29.

Clarke, A. R., Barry, R. J., McCarthy, R., \& Selikowitz, M. (2001). Excess beta in children with attention-deficit/hyperactivity disorder: an atypical electrophysiological group. Psychiatry Research, 103, 205-218.

Clarke, A. R., Barry, R. J., Dupuy, F., Heckel, L., McCarthy, R., Selikowitz, M., \& Johnstone, S. (2011). Behavioural differences between EEG-defined subgroups of children with attention-deficit/hyperactivity disorder. Clinical Neurophysiology, 122, 13331341.

Clarke, A. R., Barry, R. J., Dupuy, F. E., McCarthy, R., Selikowitz, M., \& Johnstone, S.J. (2013). Excess beta activity in the EEG of children with attention-deficit/hyperactivity disorder: a disorder of arousal? International Journal of Psychophysiology, 89, 314-319.

Conzelmann, A., Gerdes, A. B., Mucha, R. F., Weyers, P., Lesch, K. P., Bähne, C. G., Fallgatter, A. J., Renner, T. J., Warnke, A., Romanos, M., \& Pauli, P. (2014). Autonomic hypoactivity in boys with attention-deficit/hyperactivity disorder and the influence of methylphenidate. World Journal of Biological Psychiatry, 15, 56-65.

Cook, I. A., O'Hara, R., Uijtdehaage, S. H., Mandelkern, M., \& Leuchter, A. F (1998). Assessing the accuracy of topographic EEG mapping for determining local brain function. Electroencephalography and Clinical Neurophysiology, 107, 408-414.

Chabot, R., \& Serfontein, G. (1996). Quantitative electroencephalographic profiles of children with attention deficit disorder. Biological Psychiatry, 40, 951-963.

Duane, D. D. (2004). Increased frequency of rolandic spikes in ADHD children. Epilepsia, 45, 564-565.

Gordon, E., Cooper, N., Rennie, C., Hermens, D., \& Williams, L. M. (2006). Integrative neuroscience: the role of a standardized database. Clinical Electroencephalography and Neuroscience, 6, 64-75.

Holcomb, P. J., Ackerman, P. T., \& Dykman, R. A. (1985). Cognitive event-related brain potentials in children with attention and reading deficits. Psychophysiology, 22, 656-667.

Janzen, T., Graap, K., Stephanson, S., Marshall, W., \& Fitzsimmons, G. (1995). Differences in baseline EEG measures for ADD and normally achieving preadolescent males. Biofeedback Self-Regulation, 20, 65-82.

John, E. R. (1990). Principles of Neurometrics. American Journal of EEG Technology, 30, 251-266.

John, E. R. (1977). Neurometrics: Clinical Applications of Quantitative Electrophysiology. New Jersey: Lawrence Erlbaum Associates.

Jonkman, L. M., Kemner, C., Verbaten, M. N., Koelega, H. S., Camfferman, G., van der Gaag, R. J., Buitelaar, J. K., \& van Engeland, H. (1997). Event-related potentials and performance of attention-deficit hyperactivity disorder: Children and normal controls in auditory and visual selective attention tasks. Biological Psychiatry, 41, 595-611.

Johnstone, S. J., \& Barry, R. J. (1996). Auditory eventrelated potentials to a two-tone discrimination paradigm in attention deficit hyperactivity disorder. Psychiatry Research, 64, 179-192.

Kieling, C., Goncalves, R. R., Tannock, R., \& Castellanos, F. X. (2008). Neurobiology of attention deficit hyperactivity disorder. Child Adolescent Psychiatric Clinics North America, 17, 285-307.

Klorman, R., Salzman, L. F., Pass, H. L., Borgstedt, A. D., \& Dainer, K.B. (1979). Effects of methylphenidate on hyperactive children's evoked responses 
during passive and active attention. Psychophysiology, 16, 23-29.

Kopp, B., Mattler, U., Goertz, R., \& Rist, F. (1996). N2, P3 and the lateralized readiness potential in a nogo task involving selective response priming. Electroencephalography Clinical Neurophysiology, 99, 19-27.

Krause, K. H., Dresel, S. H., Krause, J., la Fougere, C., \& Ackenheil, M. (2003). The dopamine transporter and neuroimaging in attention deficit hyperactivity disorder. Neuroscience \& Biobehavioral Reviews, 27, 605-613.

Kropotov, I. D. (2009). Quantitative EEG, event related potentials and neurotherapy. San Diego: Academic Press, Elsevier.

Kropotov, I. D. (2011). Brain correlates of comparison with memory trace: Independent component analysis of event related potentials, ERPs. Key Note lecture given during $14^{\text {th }}$ International Congress of the Polish Neuropsychological Society. 24-25 October, Cracow, Poland.

Kropotov, I. D. (2014). Neuromarkers of peak performance. Doctor honoris causa Lecture. Gdansk: University of Physical Education and Sport, 8 January 2014, Gdansk, Poland.

Kropotov, I. D., Grin-Yatsenko, V. A., Ponomarev, V. A., Chutko, L. S., Yakovenko, E. A., \& Nikishena, I. S. (2005). ERPs correlates of EEG relative beta training in ADHD children. International Journal of Psychophysiology, 55, 23-34.

Kropotov, I. D., \& Mueller, A. (2009). What can event related potentials contribute to neuropsychology. Acta Neuropsychologica, 7, 169-181.

Kropotov, I. D., Chutko, L. S., lakovenko, V. A., \& Grin'-latsenko, V. A. (2002). Transcranial micropolarization in the treatment of ADHD in children and adolescents. Zhurnal nevrologii i psikhiatrii imeni S.S. Korsakova, 102, 26-28.

Makeig, S., Bell, A. J., Jung, T.-P., \& Sejnowski, T. J. (1996). Independent component analysis of electroencephalographic data. Advances in Neural Information Processing Systems, 8, 145-151.

Matousek, M., \& Petersen, I. (1973). Frequency analysis of the EEG in normal children and normal adolescents. In: P. Kellaway \& I. Petersen (eds.). Automation of clinical electroencephalography (pp. 75-102). New York: Raven.

Matousek, M., Rasmussen, P., \& Gilberg, C. (1984). EEG frequency analysis in children with so-called minimal brain dysfunction and related disorders. Advances in Biological Psychiatry, 15, 102-108.

Monastra, V. J., Lubar, J., Linden, M., Van Deusen, P., Green, G., Wing, W., Phillips, A., \& Fenger, T. (1999). Assessing attention deficit hyperactivity disorder via quantitative electroencephalography: an initial validation study. Neuropsychology, 13, 424-433.

Lubar, J. F. (1991). Discourse on the development of EEG diagnostics and biofeedback for attention-defi- cit/hyperactivity disorders. Biofeedback and SelfRegulation, 16, 201-225.

Overtoom, C. C. E., Verbaten, M. N., Kemner, C., Kenemans, J. L., van Engeland, H., Buitelaar, J. K., Camfferman, G., \& Koelega, H.S. (1998). Associations between event-related potentials and measures of attention and inhibition in the continuous performance task in children with ADHD and normal controls. Journal of American Academy of Child \& Adolescent Psychiatry, 37, 977-985.

Pascual-Marqui, R. D. (1999). Review of methods for solving the EEG inverse problem. International Journal of Bioelectromagnetism, 1, 75-86.

Pąchalska, M., Kaczmarek, B. L. J., \& Kropotov, J. D. (2014). Neuropsychologia kliniczna: od teorii do praktyki [Clinical Neuropsychology: from theory to practice]. Warszawa: Wydawnictwo Naukowe PWN.

Pąchalska, M., Kropotov, I. D., Mańko, G., Lipowska, M., Rasmus, A., Łukaszewska, B., Bogdanowicz, M., \& Mirski, A. (2012). Evaluation of a neurotherapy program for a child with ADHD with Benign Partial Epilepsy with Rolandic Spikes (BPERS) using event-related potentials. Medical Science Monitor, 18, CS94-104.

Pliszka, S. R., Liotti, M., \& Woldorff, M. G. (2000). Inhibitory control in children with attention-deficit/ hyperactivity disorder: Event-related potentials identify the processing component and timing of an impaired right-frontal response-inhibition mechanism. Biological Psychiatry, 48, 238-246.

Robaey, P., Breton, F., Dugas, M., \& Renault, B. (1992). An event-related potential study of controlled and automatic processes in 6-8-year-old boys with attention deficit hyperactivity disorder. Electroencephalography Clinical Neurophysiology, 82, 330-340.

Saletu, B., Saletu, M., \& Itil, T. (1973). The relationship between psychopathology and evoked responses before, during and after psychotropic drug treatment. Biological Psychiatry, 6, 45-71.

Satterfield, J. H., Cantwell, D. P., Lesser, L. I., \& Podosin, R. L. (1972). Physiological studies of the hyperkinetic child. American Journal of Psychiatry, 128, 102-108.

Sergeant, J. A. (2005). Modeling attention-defi cit/ hyperactivitydisorder: a critical appraisal of the cognitive-energetic model. Biological Psychiatry, 57, 1248-1255

Socanski, D., Herigstad, A., Thomsen, P. H., Dag, A., \& Larsen, T. K. (2010). Epileptiform abnormalities in children diagnosed with attention deficit/hyperactivity disorder. Epilepsy \& Behavior, 19, 483486.

Sterman, M. B. (1996). Physiological origins and functional correlates of EEG rhythmic activities: Implications for self-regulation. Biofeedback and Self-Regulation, 21, 3-33.
Diagnosis and modulation of brain dysfunctions 
Juri D. Kropotov, Maria Pąchalska, Andreas Mueller
Strehl, U., Leins, U., Goth, G., Klinger, C., Hinterberger, T., \& Birbaumer, N. (2006). Self-regulation of slow cortical potentials: a new treatment for children with attention-deficit/hyperactivity disorder. Pediatrics, 118, 1530-1540.

Thatcher, R. W., Moore, N., John, E. R., Duffy, F., Hughes, J. R., \& Krieger, M. (1999). QEEG and traumatic brain injury: rebuttal of the American Academy of Neurology 1997 report by the EEG and Clinical Neuroscience Society. Clinical Electroencephalography, 30, 94-98.

Thatcher, R. W. (1998). EEG normative databases and EEG biofeedback. Journal of Neurotherapy, 2, 8-39.

Vartanyan, G. A., Gal'dinov, G. V., Shklyaruk, S. P., Kaufman, D. A., Nikolaenko, N. N., Akimova, I. M., Trachenko, O. P., \& Novikova, T. A. (1980). Neurophysiological and structural changes at the basis of effects of transcranial micropolarization. Human Physiology, 6, 381-386.

Vendrame, M., Tracy, M., Das, R., Duffy, F., Loddenkemper, T., \& Kothare, S. V. (2010). Clinical correlations of midline spikes in children. Epilepsy \& Behaviour, 18, 460-465. 